\title{
Water-borne Use Route of Administration
}

National Cancer Institute

\section{Source}

National Cancer Institute. Water-borne Use Route of Administration. NCI Thesaurus. Code C150018.

Administration of a veterinary medicinal product to aquatic (marine or fresh water) species by medication of the water environment. 